\title{
Collège de Technologie forestière des Maritimes
}

\section{Remise de la médaille d'or de} L'IFC/CIF Gold Medal awarded

e 17 avril dernier avait lieu la $34^{\text {ème }}$

cérémonie de collation des diplômes, du Collège de Technologie forestière des Maritimes, situé à Bathurst au Nouveau-Brunswick. A cette même occasion avait lieu la remise des joncs de l'IFC aux 8 finissant(e)s du programme de technologie forestière. La médaille d'or de l'IFC/CIF a été remise cette année à Monsieur Vincent Bouchard Baker Brook, Nouveau-Brunswick. Vincent par son dévouement à son travail, son éthique personnel et professionnel incarne les principes de la médaille d'or de l'IFC/CIF. Vincent a une passion pour l'aménagement forestier, en particulier les produits forestiers non-ligneux et laménagement des lots boisés privés. Par son perfectionnisme et son énergie Vincent aura une influence majeure sur l'aménagement des forêts. Il est un grand ajout à la profession!

On April 17 the Bathurst Campus of the Maritime College of Forest Technology held its $34^{\text {nd }}$ graduation ceremony. On this occasion the CIF/IFC silver rings were presented to the eight graduating Forest Technologists. The CIF/IFC Gold Medal was presented to Vincent Bouchard of Baker Brook, New Brunswick. Vincent embodies the prin-

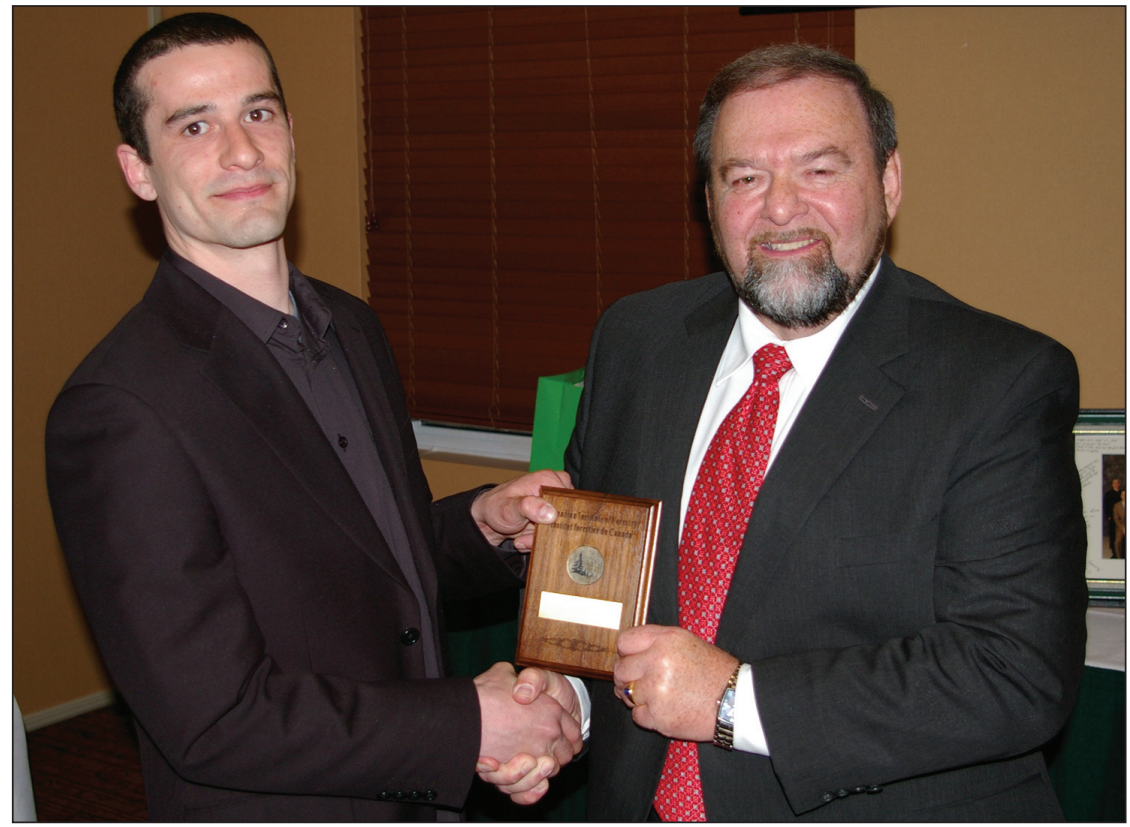

L'honorable Denis Landry, Ministre des ressources naturelles du Nouveau-Brunswick, présente la médaille d'or de l'IFC/CIF à Vincent Bouchard/The Honorable, Denis Landry, Minster of Natural Resources presents the CIF/IFC Gold Medal to Vincent Bouchard.

ciples of the gold medal. His dedication to his work, as well as his personal and professional ethics makes him an outstanding Forest Technologist. Vincent intends to pursue a career in forest management and has already begun a few projects associated with non-timber forest products and private woodlot management. Vincent, by his perfectionism, high energy level and his dedication to his work will bring great contributions to forest management and the entire forestry profession.

\section{Université de Moncton}

Médaillé d'or de l'IFC/CIF 2015 : Jonathan Pineault

Q ette année, le finissant de l'École $\checkmark$ de foresterie (ÉdeF) de l'Université de Moncton, campus d'Edmundston (UMCE), Jonathan Pineault, s'est mérité la Médaille d'or de l'Institut forestier $d u$ Canada/Canadian Institute of Forestry ('IFC/CIF). Originaire de Val-d'Or, au Québec, Jonathan sest distingué tout au long de ses études universitaires par son rendement académique, ainsi que par son engagement et son implication à
l'Université. En 2012, Jonathan a complété ses premières études en foresterie dans le programme de technologie forestière au Cégep de l'Abitibi-Témiscamingue à Rouyn-Noranda. Durant ce programme, il a mérité des bourses dexcellence pour ses résultats académiques et, à la fin, la Médaille du mérite forestier.

À l'automne 2012, il a débuté son programme de Baccalauréat en sciences forestières à l'École de foresterie de I'UMCE. Dès sa première année à cette institution, il sest impliqué activement dans les activités étudiantes et aussi dans la communauté en tant que bénévole. Entre autres, en 2013, il était bénévole dans le cadre de la conférence internationale sur la sylviculture des feuillus de l'Institut de recherche sur les feuillus nordiques et, en 2014, à la cérémonie de fermeture du Congrès Mondial Acadien et au souper bénéfice du Quality Deer Management Association. En 2014-2015, il occupait le poste de vice-président au sein du Conseil étu- 
diant de l'ÉdeF. Durant cette année, le Secteur sciences forestières a été choisi le secteur de l'année à l'UMCE. Cet honneur est remis annuellement à un secteur étudiant de l'Association générale des étudiants et étudiantes (AGÉÉ) de l'UMCE qui sest démarqué par la mise en oeuvre d'activités créatives autant sur le Campus quà l'extérieur en créant un climat de rapprochement entre les employés du secteur et les étudiantes et étudiants. De plus, les membres du Conseil ont su démontrer un appui remarquable et soutenu aux activités de l'AGÉÉ.

Jonathan a profité de ses étés pour acquérir de l'expérience de travail en foresterie, et ce dans des domaines très diversifiés. Il a travaillé comme stagiaire avec la Pourvoirie Monet à Val-d'Or, et comme stagiaire et technologue forestier pour le Ministère des Ressources naturelles et le Ministère des Forêts, de la Faune et des Parcs à différentes endroits au Québec. Entre autres, il a été responsable de lélaboration de plans de sondages, la compilation de données terrain, la prospection du territoire pour des travaux sylvicoles, et le suivi de chantiers de récolte et du Manuel d’aménagement forestier du Québec.

En ce qui concerne sa carrière future, il a un intérêt marqué pour l'amélioration des procédés, la gestion de projets et la planification des opérations forestières.

\section{Remise des Joncs de l'IFC/CIF}

Cette année, cinq finissantes et finissants de l'École de foresterie de l'Université de Moncton, campus d'Edmundston, méritaient leur jonc de forestier de l'Institut forestier du Canada/Canadian Institute of Forestry (l'IFC/CIF). Il sagit de Maxime Auger, Saint-Raymond, Québec, Zlatko Blazeski, Saint-Nicéphore, Québec, Stéphanie Boulianne, Saint-Eugène-deChazel, Québec, Sophie Gosselin, Dieppe, N.-B. et Jonathan Pineault, Vald’Or, Québec.

La remise des joncs est gérée par l'IFC/CIF depuis 1967. Dans le cadre de cette activité, les finissantes et finissants des sept universités canadiennes offrant

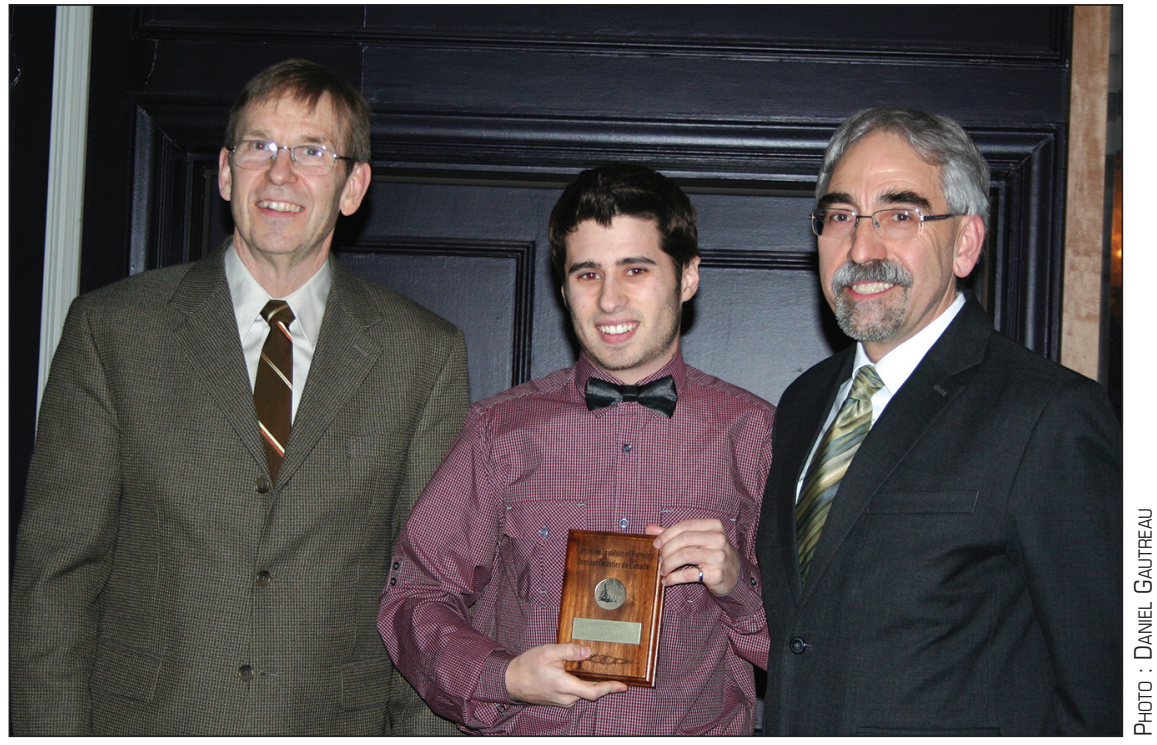

De gauche à droite : Al Stinson, président Institut forestier du Canada ; Jonathan Pineault, médaillé d'or de l'IFC/CIF ; Roger Roy, directeur École de foresterie

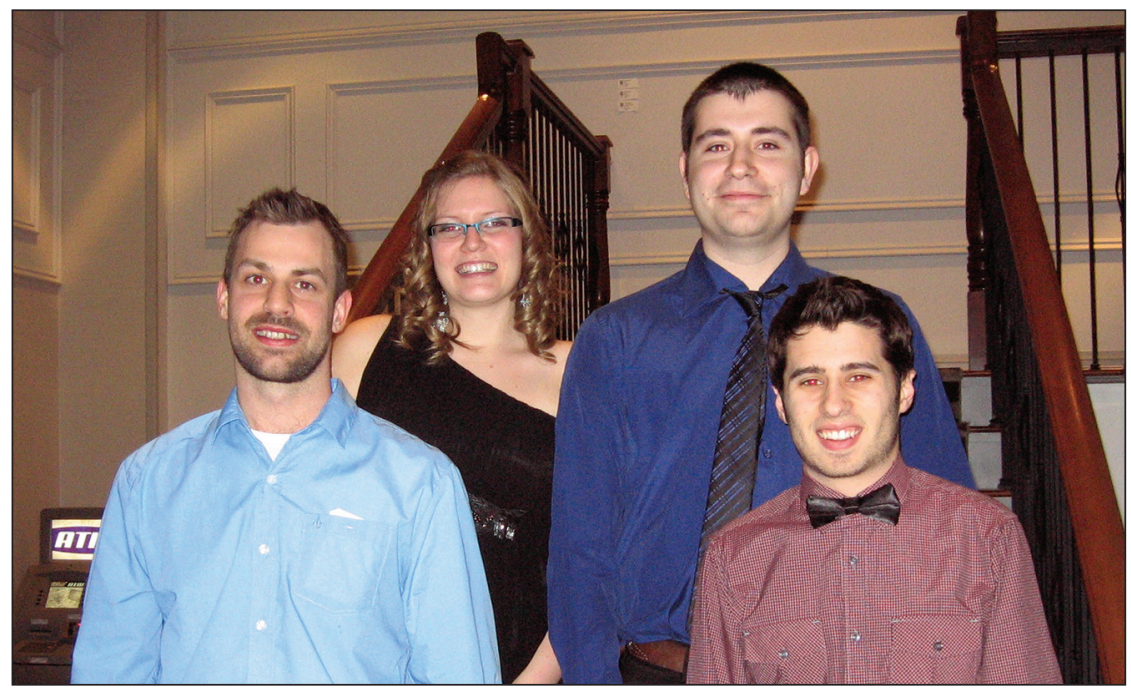

Gauche à droite : Maxime Auger, Stéphanie Boulianne, Zlatko Blazeski, Jonathan Pineault (absente au moment de la photo : Sophie Gosselin)

un baccalauréat dans plusieurs domaines reliés à la foresterie reçoivent un jonc identique signalant leur appartenance au groupe diversifié des professionnels de la forêt. Cette année, la remise des joncs aux finissantes et finissants de l'École de foresterie de l'Université de Moncton, Campus d’Edmundston, ainsi quà celles et ceux de la Faculty of Forestry and Environmental Management de la University of New Brunswick, avait lieu dans le cadre d'une cérémonie organisée par l'IFC/CIF qui a eu lieu le 25 février dernier à Fredericton. 


\section{La Cité Collégiale}

U ne tradition s'est poursuivie à La Cité pour les finissants du programme de Techniques en environnement forestier alors que ceux-ci ont récemment reçu leur jonc d'argent de l'Institut forestier du Canada/Canadian Institute of Forestry.

Des représentants de l'organisme national étaient de passage à La Cité pour y remettre les joncs aux finissants, de même qu'une médaille d’or à Josée Séguin, qui a maintenu d'excellents résultats scolaires tout en étant activement impliquée dans la vie étudiante. Les finissants de La Cité ont par la même occasion eu droit à quelques conseils de la part des représentants de l'Institut forestier du Canada/Canadian Institute of Forestry, dont André Rousseau, conseiller de la section Vallée de l'Outaouais.

« Nous vous prions de le porter avec fierté et humilité. Il représente votre investissement en vous-même. Il symbolise votre engagement à agir de façon éthique et avec professionnalisme ", a souligné $\mathrm{M}$. Rousseau au sujet du jonc que recevait chaque finissant.
«Saisissez toutes les opportunités qui vous sont offertes. Ces occasions pourraient vous mener loin dans vos carrières. Deuxièmement, continuez à développer vos compétences en communications. C'est une qualité très recherchée par les employeurs ", a pour sa part indiqué Michael Rosen, président d’Arbres Canada et directeur de la section Vallée de l'Outaouais de l'Institut forestier du Canada/Canadian Institute of Forestry.

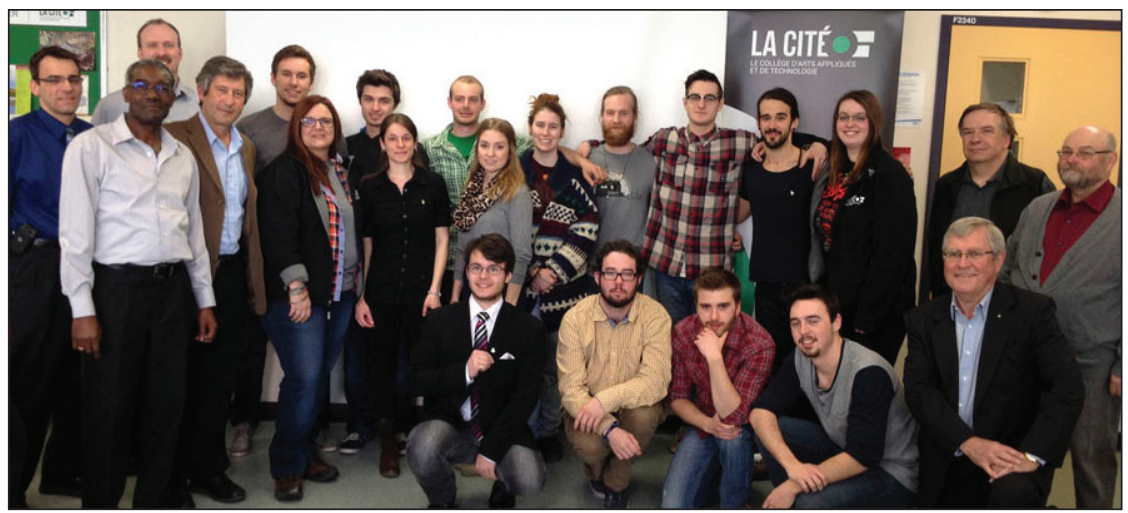

Les finissants en environnement forestier voient leurs efforts récompensés.
Remis pour la première fois à l'Université de la Colombie-Britannique en 1953, les joncs forestiers symbolisent la fin des études au sein d'un programme de foresterie reconnu par l'Institut forestier du Canada/Canadian Institute of Forestry. Ils sont décernés annuellement à des diplômés d'un bout à l'autre du pays.

\section{Cégep de Saint-Félicien}

V alérie-Savard-Bergeron, finissante en Techniques du milieu naturel de la voie de spécialisation "Aménagement de la ressource forestière ", a obtenu la Médaille d'or de l'Institut forestier du Canada/Canadian Institute of Forestry pour le Cégep de Saint-Félicien.

Valérie est originaire de St-Félicien. Personne dynamique et consciencieuse, en plus de son rôle de jeune maman, elle a su se distinguer par son sens critique et sa connaissance du milieu forestier. Nul ne doute qu'elle sera un atout important dans son milieu de travail.

Chaque année, l'Institut remet cette 